\title{
Fibrotic content of LV myocardium quantified by CMR characterizes left atrial sizes and total left atrial emptying function incremental to LV functional parameters and LV myocardial mass index in patients with hypertrophic cardiomyopathy
}

\author{
Yucheng Chen ${ }^{1 *}$, Carolyn Ho ${ }^{1}$, Eri Watanabe ${ }^{1}$, Damien Mandry ${ }^{1}$, Michael Jerosch-Herold ${ }^{2}$, Raymond Kwong ${ }^{1}$
}

From 15th Annual SCMR Scientific Sessions

Orlando, FL, USA. 2-5 February 2012

\section{Summary}

Fibrotic burden of LV myocardium quantified by CMR provides additional information about LA size and mechanics than morphological severity of LV hypertrophy measured by LV mass index alone.

\section{Background}

Progressive myocardial stiffness of the LV in patients with hypertrophic cardiomyopathy (HCM) impedes early diastolic filling and left atrial (LA) emptying functions. Over time, LA of patients with HCM progressively dilates and undergoes structural and functional remodeling with serious cardiac events such as development of atrial fibrillation and diastolic heart failure despite optimal medical therapy.

\section{Methods}

We studied $65 \mathrm{HCM}$ patients (46 men, mean age 39 \pm 14 years, mean LVEF $63 \pm 9 \%$ ) with CMR. The fibrotic content of the LV myocardium (FIMeanSeg, averaged over 18 segments) was obtained using serial R1 mapping by cine-IR method before and up to 30 minutes after contrast injection. LA volumes and LA emptying function across 3 phases of the cardiac cycles, and LV function were also quantified. We sought to determine the strongest variables that predispose the patients to have LA dilatation and impaired LA emptying function.

\section{Results}

FIMeanSeg demonstrated strong positive correlation with LA volumes across all 3 phases of the cardiac cycle, with the strongest correlation with LAMax volume at end-ventricular systole $(\mathrm{r}=0.41, \mathrm{P}=0.0005$, Figure 1). FIMeanSeg also demonstrated significant negative correlations with total and contractile LA emptying functions $(r=-0.25$ and $-0.27, \mathrm{P}=0.04$ and 0.02 , respectively). Stepwise linear regression selected FIMeanSeg with LVEDV and LVESV as the best-overall model for LA size (Table 1). FIMeanSeg was the strongest multivariable covariate in the best-overall model for total LA emptying function, with substantial incremental value over LV mass index (Table 1).

\section{Conclusions}

FIMeanSeg provides independent association with LV volume beyond knowledge of ventricular functional parameters and severity of ventricular hypertrophy by LV mass. This novel method may add to the current characterization of impaired diastolic function in HCM patients and may have prognostic implications.

${ }^{1}$ Cardiology Division, Brigham and Women's Hospita; Boston, MA, USA

Full list of author information is available at the end of the article

(C) 2012 Chen et al; licensee BioMed Central Ltd. This is an open access article distributed under the terms of the Creative Commons Attribution License (http://creativecommons.org/licenses/by/2.0), which permits unrestricted use, distribution, and reproduction in any medium, provided the original work is properly cited. 


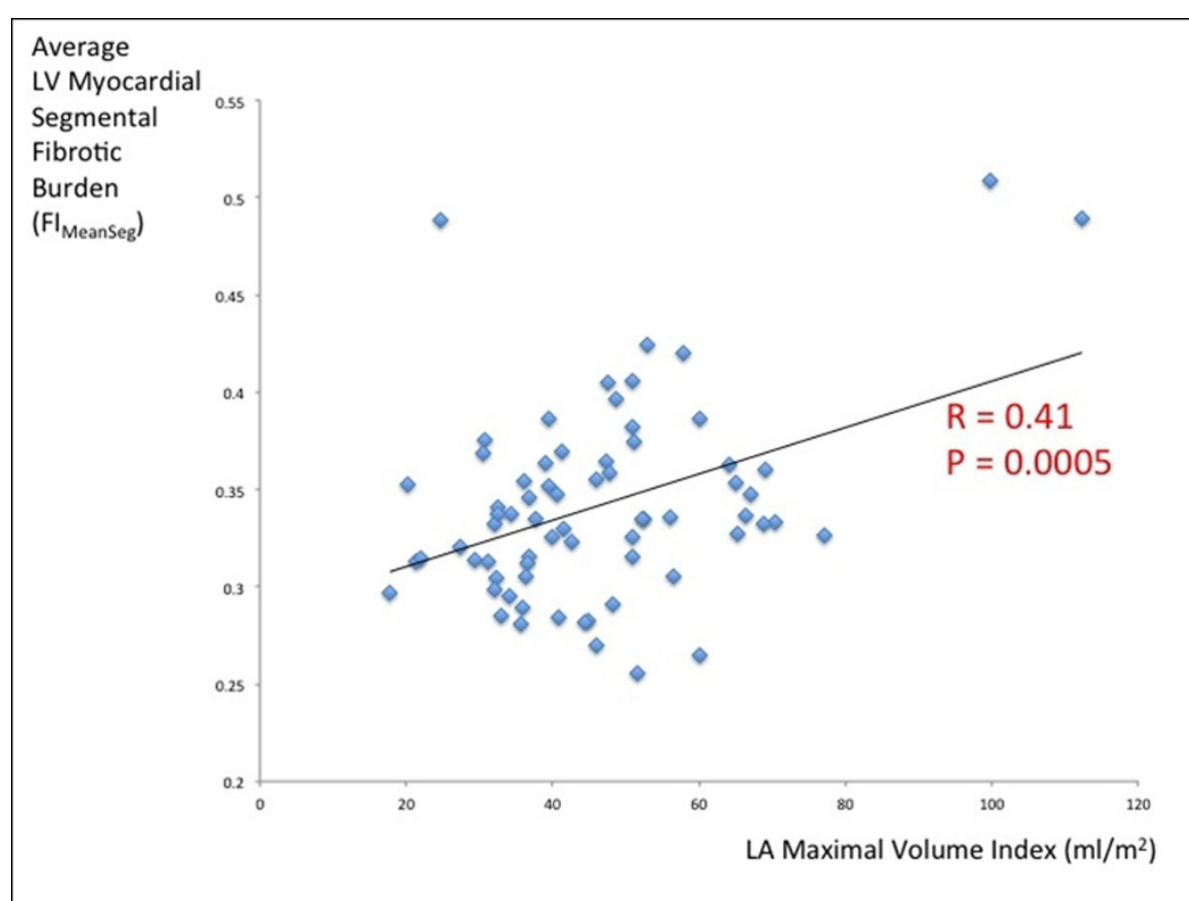

Figure 1

Table 1 Correlation between LV fibrotic index and left atrial size or function

\begin{tabular}{ccc}
\hline \multicolumn{3}{c}{ Best Overall Model for Maximal Left Atrial Size } \\
\hline & F-value & P-value \\
\hline FIMeanSeg & 13.22 & 0.0006 \\
LVEDV $(\mathrm{ml})$ & 6.34 & 0.01 \\
LVESV $(\mathrm{ml})$ & 18.89 & 0.0002 \\
\hline \hline Best Overall Model for Total Left Atrial Emptying & Function \\
\hline & F-value & P-value \\
\hline FIMeanSeg & 8.26 & 0.0055 \\
LV mass index $(\mathrm{g} / \mathrm{m} 2)$ & 6.50 & 0.01 \\
\hline
\end{tabular}

\section{Funding}

Yucheng Chen got the salary from China Scholarship Council.

\section{Author details}

${ }^{1}$ Cardiology Division, Brigham and Women's Hospita; Boston, MA, USA.

${ }^{2}$ Radiology Department, Brigham and Women's Hospital, Boston, MA, USA.

Published: 1 February 2012

doi:10.1186/1532-429X-14-S1-P159

Cite this article as: Chen et al:: Fibrotic content of LV myocardium quantified by CMR characterizes left atrial sizes and total left atrial emptying function incremental to LV functional parameters and LV myocardial mass index in patients with hypertrophic cardiomyopathy. Journal of Cardiovascular Magnetic Resonance 2012 14(Suppl 1):P159.
Submit your next manuscript to BioMed Central and take full advantage of:

- Convenient online submission

- Thorough peer review

- No space constraints or color figure charges

- Immediate publication on acceptance

- Inclusion in PubMed, CAS, Scopus and Google Scholar

- Research which is freely available for redistribution 